\title{
Mesenchymal stem cells in cancer progression and anticancer therapeutic resistance
}

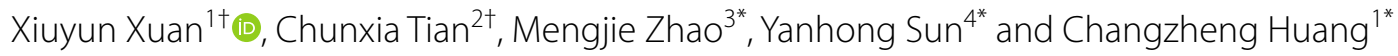

\begin{abstract}
Increasing evidence indicates that the tumor microenvironment appears to play an increasingly important role in cancer progression and therapeutic resistance. Several types of cells within the tumor stroma had distinct impacts on cancer progression, either promoting or inhibiting cancer cell growth. Mesenchymal stem cells (MSCs) are a distinct type of cells that is linked to tumor development. MSCs are recognized for homing to tumor locations and promoting or inhibiting cancer cell proliferation, angiogenesis and metastasis. Moreover, emerging studies suggests that MSCs are also involved in therapeutic resistance. In this review, we analyzed the existing researches and elaborate on the functions of MSCs in cancer progression and anticancer therapeutic resistance, demonstrating that MSCs may be a viable cancer therapeutic target.
\end{abstract}

Keywords: Mesenchymal stem cells, Cancer progression, Therapeutic resistance, Tumor microenvironment, Cytokine

\section{Background}

Mesenchymal stem cells (MSCs) are a kind of multipotent cells with a high differentiation potential and selfrenewal ability, making them a promising regenerative medicine population [1]. MSCs are easy to acquire and may be separated from a wide range of tissues. The major source of MSCs is believed to be bone marrow (BM), although the content is minimal, accounting for just $0.001-0.01 \%$ of total nucleated cells [2]. In the BM, MSCs support haemopoiesis and regulate immune activity [3]. Moreover, MSCs may also be isolated from adipose

\footnotetext{
*Correspondence: mengjieclinic@whu.edu.cn; sunyh1128@zju.edu.cn; d202081737@hust.edu.cn

${ }^{\dagger}$ Xiuyun Xuan and Chunxia Tian contributed equally to this work

${ }^{1}$ Department of Dermatology, Union Hospital, Tongji Medical College,

Huazhong University of Science and Technology, Wuhan 430022, Hubei, China

${ }^{3}$ Department of Dermatology, Zhongnan Hospital, Wuhan University, Wuhan 430022, Hubei, China

${ }^{4}$ Department of Dermatology, Second Affiliated Hospital, Zhejiang University School of Medicine, Hangzhou 310009, Zhejiang, China

Full list of author information is available at the end of the article
}

tissue, which comes from people who have had liposuction. Adipose tissue-derived stem cells serve a crucial role in reconstructive or tissue engineering medicine [4]. In addition to the two sources of MSCs mentioned above, MSCs can be also effectively extracted from other tissues, such as umbilical cord [5], umbilical cord blood [6], amniotic membrane [7], placenta [8], peripheral blood [9], muscle [10] and lung [11]. MSCs have a variety of functions in these organs, including contributing to organ homeostasis and tissue-specific healing [12]. In addition, MSCs have three basic characterizations. To begin, morphological characterization of MSCs revealed that they are a heterogeneous cell population with numerous cell subsets that are spindle-form fibroblastlike, flattened, or spherical in shape [13-15]. Second, the functional and differentiation characteristics of MSCs indicated that, when subjected to the appropriate stimuli, they may differentiate into a number of cell types (such as fibroblasts, adipocytes, chondrocytes and osteoblasts) and perform a variety of roles [16]. Third, immunophenotypic characterization of MSCs exhibited that they original author(s) and the source, provide a link to the Creative Commons licence, and indicate if changes were made. The images or other third party material in this article are included in the article's Creative Commons licence, unless indicated otherwise in a credit line to the material. If material is not included in the article's Creative Commons licence and your intended use is not permitted by statutory regulation or exceeds the permitted use, you will need to obtain permission directly from the copyright holder. To view a copy of this licence, visit http://creativecommons.org/licenses/by/4.0/. The Creative Commons Public Domain Dedication waiver (http://creativeco mmons.org/publicdomain/zero/1.0/) applies to the data made available in this article, unless otherwise stated in a credit line to the data. 
express the cell surface markers CD105, CD90 and CD73 but lack CD45, CD34, CD14, CD11b, CD79a or CD19, as well as HLA-DR [17]. Moreover, MSCs are also said to have immune-suppressive properties [1]. MSCs modulate immunity by producing cytokines and regulating several immune cells' function [18]. For example, MSCs have been shown to be effective in treating graft disease versus host disease [19] and some autoimmune diseases [20]. In addition, MSCs also have the ability to migrate to tumor and inflammatory areas. Several chemokines and related receptors may be involved in the process of MSC migration, including growth factors (i.e., EGF, SCF, PDGF, HGF and IGF-1 [21, 22]), angiogenic factors (i.e., VEGF, HIF $1 \alpha$ and $\beta F G F[23,24]$ ), chemokines (i.e., CCL2, CCL5, CCL22 and CXCL12 $[22,25])$, inflammatory factors and other cytokines (i.e., TNF $\alpha$, TGF $\beta$, IL-1 $\beta$, IL- 8 [26-29]). Recently, it is reported that cancer cell-derived exosomes (Exo) have been shown to regulate the migration and homing of MSCs by inducing the expression of circular RNAs. In gastric cancer, for example, after MSCs were treated with adenocarcinoma gastric cells cell-derived Exo, elevated hsa_circ_0004303 in MSCs promoted the biological activities of MSCs via the miR148a-3P/ALCAM axis [30]. These findings showed that a variety of mediators may aid MSC migration to various types of tumor locations, therefore promoting or inhibiting tumor growth. Because of their homing ability, MSCs have been successfully utilized to treat spinal cord injury, damaged kidneys, diabetes, myocardial infarction, and bone injury [17]. Furthermore, MSCs have been shown to have the capacity to stimulate angiogenesis during the process of ischemia [31] and wound healing [32]. In summary, MSCs perform a variety of vital functions under physiological and pathological circumstances, and play crucial roles in the treatment of several diseases. In addition, MSCs have been indicated to also have a positive or negative effect on cancer pathogenesis.

In this review, we gathered related researches and discussed the functions of MSCs in cancer progression and anticancer therapeutic resistance, which may provide potent targets for cancer and find new improvements for therapeutic resistance.

\section{MSCs in cancer progression}

Tumor microenvironment (TME) heterogeneity is a significant component that impacts tumorigenesis. TME is a complex ecosystem that comprises stromal cells, extracellular matrix components, and secreted factors [33]. Among of them, stromal cells in TME incorporate that adipocytes, endothelial cells, cancer-associated fibroblasts, immune cells and MSCs [34]. MSCs, in particular, have a strong tropism to tumor locations, accelerating or slowing cancer growth. However, the exact mechanism is not clear. Then we will discuss the role of MSCs in cancer pathogenesis.

\section{MSCs promote cancer progression}

TLRs (Toll-like Receptors) are found on a variety of cell types, including MSCs. TLRs have the capacity to detect "danger" signals, and their activation attracts a variety of cells to the damaged region, including immune cells and MSCs. Interestingly, TLR3 activation resulted in MSCs secreting some factors with mostly tumor supportive immunosuppressive effect (such as IL1RA and IL10), while TLR4 stimulation leaded to MSCs producing inflammatory and proapoptotic factors (such as IL17, GM-CSF, and TRAIL). TLR4-primed MSCs are called MSC1 that exhibited an antitumorigenic impact, while TLR3-primed MSCs are called MSC2 that had a tumorsupportive function [35]. In addition, according to Ruth and colleagues, MSC1 inhibits tumor growth while MSC2 increases tumor growth and metastasis in vivo and in vitro [36]. Interestingly, the type of TLR agonist exposure to MSCs help the switch between MSC1 and MSC2. In other words, TLR4 agonists polarize MSCs towards pro-inflammatory MSC1 which is crucial for early injury responses, whereas TLR3 agonist exposure will polarize MSCs towards immunosuppressive MSC2 which is necessary for helping to heal tissue injury. Maybe it can help explain why MSCs have diverse roles in various cancer types.

Notably, MSCs have been shown to inhibit the antitumor immune response, including innate and adaptive immune responses, by secreting a variety of soluble factors and mediators (such as prostaglandin E2 (PGE2), IFN $\gamma$, IL-4, indoleamine 2,3-dioxygenase (IDO), TGF$\beta 1$, IL-6) and interacting with diverse immune cell types (such as T cell, B cells, macrophages, dendritic cells, NK cells and neutrophils) [37]. In adaptive immune responses, MSCs reduced $\mathrm{T}$ cell activation and proliferation. MSCs secreted PGE2, then bind to prostaglandin EP2 and EP4 receptors to reprogram macrophages by producing anti-inflammatory factor IL-10, which thus inhibit T cells [38]. Moreover, MSCs elicited Th2polarized immune response. In other words, inflammatory $\mathrm{T}$ cells and associated cytokines (Th1 cells-IFN $\gamma$ ) were reduced, whereas anti-inflammatory $\mathrm{T}$ cells and related cytokines (Th2 cells-IL4) were elevated [39]. In addition, MSCs have been shown to block $\mathrm{T}$ cell activation by releasing immunosuppressive TGF- $\beta 1$, which bind to glycoprotein a repetitions predominant (GARP) expressed on MSCs [40]. Furthermore, through degrading tryptophan, IDO produced by MSCs was able to suppress allogeneic T-cell responses [41]. Notably, in naive $\mathrm{CD}^{+} \mathrm{T}$ cells, tryptophan catabolism triggered the development of FOXP3-positive regulatory $\mathrm{T}$ cells 
(Treg) [42]. These cells reduced anti-tumor immunity by suppressing effector $\mathrm{T}$ cell responses. Recently, a novel mechanism by which MSCs regulate the immune system was discovered. It is that MSCs recruited myeloidderived suppressor cells (inhibitory immune cells) in a CCL2-dependent way, reducing anti-cancer $\mathrm{T}$ cell activity even more [43]. In addition to $\mathrm{T}$ cells in adaptive immune response, MSCs can also suppress B cell functions. MSCs produced humoral substances that inhibited B cell activity by suppressing B cell terminal differentiation [44]. IFN $\gamma$-activated MSCs increased the expression of galectin-9, which inhibited antigen triggered immunoglobulin release and slowed B cell proliferation [45]. Taken together, MSCs have strong inhibitory effects on adaptive immune response, which is extensively exploited by cancer cells within TME. In addition to suppression of adaptive immune response, MSCs also inhibited innate immune cells to weaken primary anti-cancer immune responses. PGE2 and IL-6 produced by MSCs suppressed NK cell functions. And, MSCs primarily inhibited IFN- $\gamma$ production in NK cells, thus weakening their anti-cancer activity [46]. Moreover, anti-cancer activities are inextricably linked with dendritic cells (DCs), which act to present antigens. It has been shown that DCs maturation and function were inhibited in the presence of PGE2 generated by MSCs [47]. And, MSCs suppressed development and function of monocyte-derived DCs with costimulatory molecules CD80/CD86 expression reduced, limiting allogeneic $\mathrm{T}$ cell allostimulatory capacity [48]. In addition, within the TME, macrophage activity was reduced directly by MSCs. MSC-derived conditioned medium (CM) has been shown to impair macrophage phagocytic activity, further decreasing anti-cancer immunity [49]. And, MSC-derived PGE2 induced a transition from inflammatory M1 macrophages to a pro-tumorigenic alternatively activated M2 state, which was accompanied by increased levels of immune-inhibitory IL-10 [50]. Furthermore, neutrophils activity was also influenced by MSCs. In breast tumor model, CD11b ${ }^{+} \mathrm{Ly} 6 \mathrm{G}^{+}$neutrophils were trained to acquire immunosuppressive activity following coculture with MSCs, suppressing T cell proliferation in vitro, and enhancing tumor progression in vivo [51]. Similarly, in gastric cancer, the chemotaxis, survival and activation of neutrophils were regulated via IL6STAT3-ERK1/2 signaling, thus supporting tumor progression [52]. Taken together, these data above indicated that MSCs were able to suppress the anti-tumor immune response, therefore prompting tumor growth.

Moreover, MSCs were able to stimulate cancer cell growth and angiogenesis. In breast and prostate tumors, for example, MSCs raised the amounts of pro-angiogenic factors, such as MIP-2, VEGF, TGF- $\beta$ and IL-6. These factors induced tumor cells proliferation and angiogenesis, thereby increasing the pace of solid tumor development in vitro and in vivo [53]. Similarly, in hepatocellular carcinoma, Li et al. discovered that the level of microvessel density and TGF $\beta 1$ mRNA were considerably enhanced, but Smad7 mRNA expression was inhibited in the MSC treated group. Their study indicated that MSCs may stimulate angiogenesis via the TGF $\beta 1 /$ Smad pathway [54]. Recently, Yuan et al. discovered that LncRNA H19 is implicated in MSC-mediated angiogenesis [55]. They found that LncRNA H19 knockdown in MSCs suppressed angiogenesis by associating with histone methyltransferase EZH2 and activating the angiogenesis inhibitor gene VASH1, reducing angiogenesis factors secretion and increasing angiogenesis inhibitors production.

In addition, MSCs promoted cancer cells metastasis, thus hastening tumor development. When breast cancer cells were directly co-cultured with MSCs, they demonstrated substantial overexpression of oncogenes (NCOA4, FOS), proto-oncogenes (FYN, JUN), and EMT specific markers, as well as shape and growth pattern changes, resulting in breast cancer metastasis [56]. Notably, cancer stem cells (CSCs) are particularly important in tumor metastasis. MSCs were indicated to prompt CSCs proliferation by producing several tumor-supportive mediators, thus making tumor spread and growth easier [57]. In addition, mesenchymal niche may be involved in cancer metastasis. Emerging evidences suggested that MSCs can move to tumor locations, including primary and pre-metastatic sites [58]. Tumor-secreted factors may go to neighboring tissues [59], where they attract MSCs to help build up the mesenchymal niche, which encourages cancer cell migration. In breast cancer, cancer cells induce production of CCL5 (also called RANTES) from MSCs via interacting with CCR5, increasing cancer cell motility, invasion, and metastasis in vitro and in vivo [60].

Furthermore, MSCs were also able to prevent tumor cells from undergoing apoptosis. As is all known, hypoxia, malnutrition, and inflammation are all recognized to have roles in tumor pathogenesis. MSCs sustain their self-survival in these settings via autophagy and the release of numerous pro-survival or anti-apoptotic factors, such as VEGF, bFGF, PDGF, TGF- $\beta$, SDF- $1 \alpha$, HGF, and Nitric oxide (NO) [61]. VEGF and bFGF, for example, can boost Bcl-2 expression [62, 63], while PDGF and TGF- $\beta$ can boost VEGF and bFGF gene expression [64]. SDF-1 $\alpha$ has been shown to protect leukemia cells against spontaneous apoptosis [65]. And, HGF improved the angiogenic and anti-apoptotic effects [66]. Moreover, NO was thought to be a dual-function apoptotic regulator. In short, at large doses, NO is proapoptotic, whereas at low doses, it is antiapoptotic [67]. 
Additionally, MSCs support tumor growth by changing their metabolic state. In lymphoblastic leukemia, MSCs-derived PGE2 activated cAMP-PKA signaling in tumor blasts and inhibited the tumor-suppressive function of wild type p53, thus promoting leukaemogenesis [68]. When exposed to oxidative stress in the TME, MSCs can produce lactate, and when cancer cells absorb lactate, their migration is enhanced by producing ATP [69]. Particularly, MSCs have been observed to differentiate into CAFs in vitro, which could drive tumor heterogeneity and play a crucial role in cancer progression and drug resistance [70]. In addition, increasing evidence has shown that noncoding RNAs participate in tumorigenesis and drug resistance [71-73]. A recent study showed that in gastric cancer, TGF- $\beta 1$ secreted by MSCs activated the SMAD2/3 pathway and supported cancer progression through the lncRNA MACC1-AS1/miR-145-5p/ fatty acid oxidation (FAO) axis in cancer cells [74]. Moreover, in triple-negative breast cancer, MSCs strongly induced the regulation of LINC01133 in adjacent tumor cells, which increases the propagation of CSC-like phenotypic characteristics and therefore strengthens cancer cell growth [75].

These findings revealed that MSCs support cancer progression via distinct mechanisms (Table 1; Fig. 1). Targeting MSCs represents a potential strategy for the treatment of cancer.

\section{MSCs inhibit cancer progression}

Notwithstanding the effects of MSCs on cancer progression, studies have demonstrated their inhibitory effect on tumor growth. As a proinflammatory factor, the combination of MSCs and tumor cells increased the infiltration of monocytes, granulocytes as well as $\mathrm{T}$ lymphocytes. The increased infiltration of inflammatory cells creates opportunities for cross-talk between these immune cells and the surrounding tissues. These immune cells, as well as the surrounding inflamed tissues, can generate several chemokines that attract activated lymphocytes with the corresponding receptors, thereby further inducing anticancer immunity [76]. Furthermore, Aarif and colleagues showed that MSCs suppressed tumor growth in vivo by inhibiting target cell AKT activity in Kaposi's sarcoma (KS). However, they discovered that when KS tumor cells were engineered to express active AKT continuously, KS tumors were no longer sensitive to MSCs administration. Their findings suggest that MSCs exert potent antitumorigenic effects by inhibiting AKT signaling [77]. Similarly, Qiao et al. demonstrated that MSCs suppress breast cancer cell proliferation via the Wnt pathway, which is crucial in tumourigenesis [78] (Fig. 1). In addition, in a study by Lu and colleagues, they showed that MSCs administration enhanced the mRNA expression of p21 (cell cycle negative regulator) and caspase 3 (apoptosis associated protease) in tumor cells. Their findings demonstrated that MSCs can inhibit cancer progression in vitro and in vivo by increasing apoptosis and G0/G1 phase arrest in cancer cells [79]. Furthermore, MSCs have been shown to suppress cancer by inhibiting tumor angiogenesis through endothelial cells apoptosis and capillary degeneration [80]. Recently, Gu and colleagues reported that MSCs-derived Exo were able to block hepatocellular CSCs malignancy via a lncRNA C5orf66-AS1/ microRNA-127-3p/dual-specificity phosphatase 1 (DUSP1)/ ERK axis [81]. Considering that Exo are involved in both oncogenic and tumor-suppressing roles of MSCs, they treated hepatocellular CSCs with MSC-Exo, and found that the proliferation, migration, invasion, angiogenesis-stimulating and self-renewal abilities of CSCs were significantly decreased through lncRNA C5orf66-AS1/ microRNA-127-3p/DUSP1 axis and inhibiting the phosphorylation of ERK in vitro. And in vivo, similar results were observed, which showed that Exo attenuated the growth of xenograft formed by CSCs in nude mice. Their study may offer novel insights into the relevance of MSCs and their derived molecules to cancer progression, and in particular, to the stem cell property of CSCs (Table 1).

Alternatively, engineered MSCs are gaining popularity because of their propensity to migrate to tumor locations. For example, TNF-related apoptosis-inducing ligand (TRAIL), nanoparticles, bone morphogenetic protein 4 (BMP4) and other molecules that can limit cancer cell development were used for modifying MSCs, which reduced cancer cell growth and metastasis while also causing apoptosis [82-84]. More molecules were administered to engineered MSCs, as shown in Table 3. These findings indicated that modified MSCs can reduce the proliferation and migration of cancer cells in vitro and in vivo, implying that MSCs could become a potential therapeutic for cancer.

\section{MSCs in anticancer therapeutic resistance}

Resistance to therapeutic therapy leads to the limited efficacy of cancer treatments. Although emerging evidence indicates that tumor cell-intrinsic gene alterations contribute to therapeutic resistance, an increasing number of reports have suggested that the TME is also crucial in the evolution of therapeutic resistance [85]. Intrinsic resistance develops over time, while external resistance can be mediated by signaling or soluble molecules from the TME. The latter resistance may be reversible because therapeutic sensitivity was restored when these TME mediators were removed [85]. Thus, it is crucial to understand the mechanism of external therapeutic resistance. Notably, MSCs, which are an important part of the TME, 


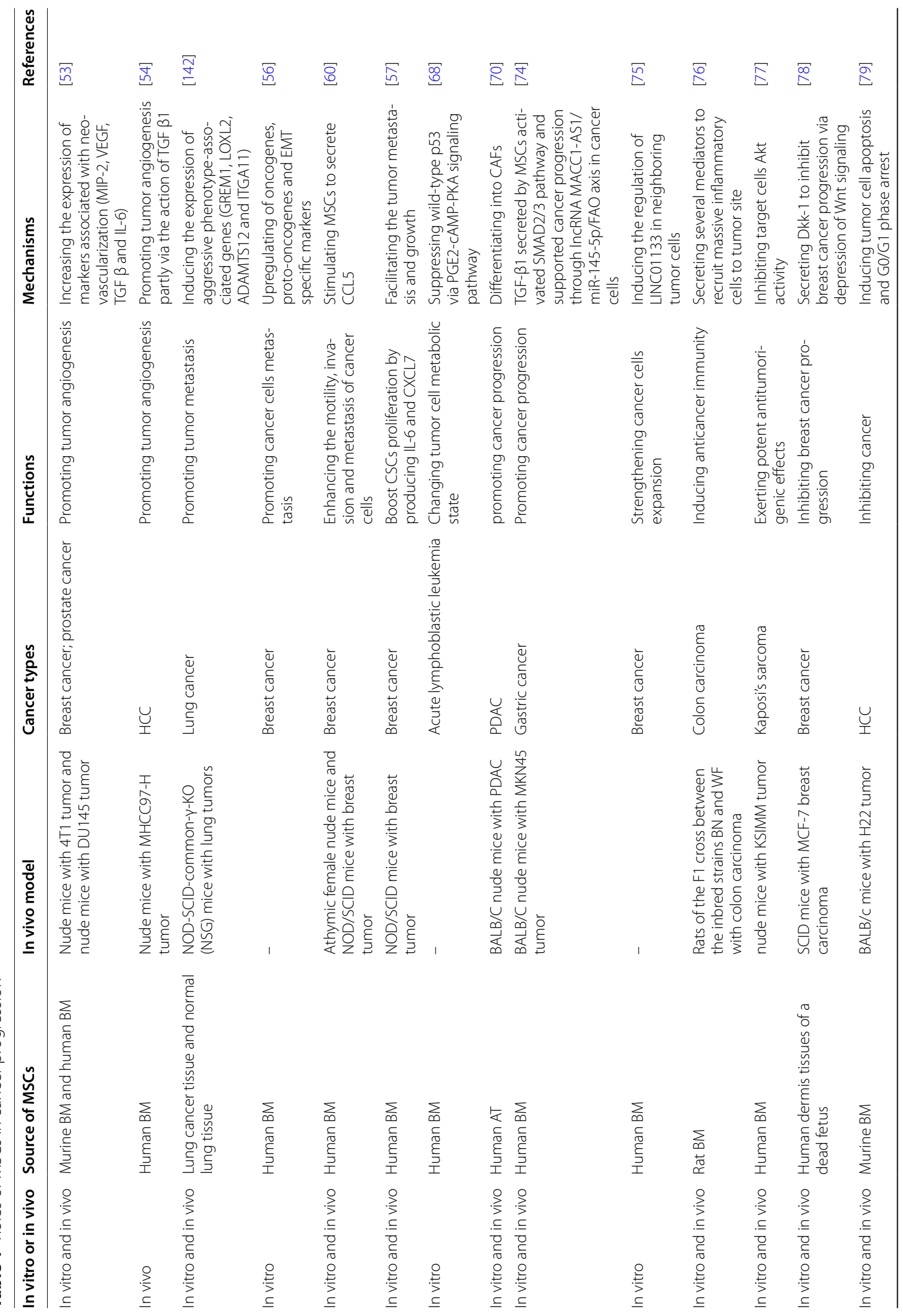




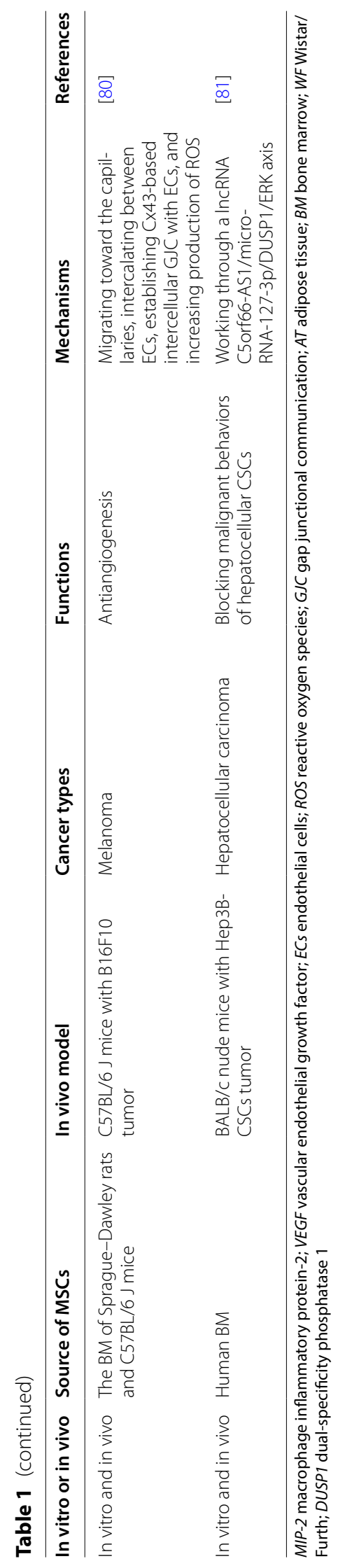




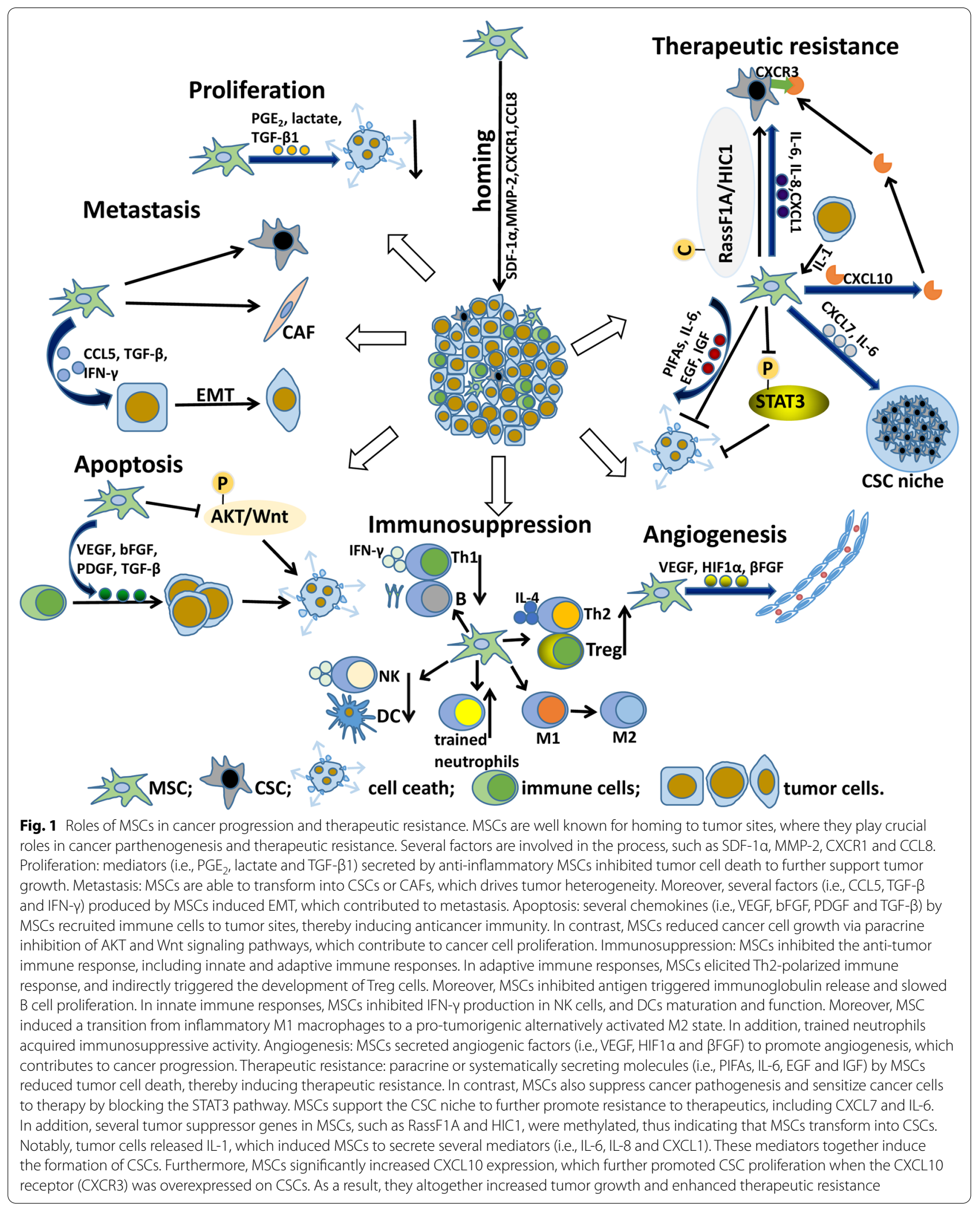


are known to play a key role in the resistance of cancer treatment [86].

\section{MSCs promote therapeutic resistance}

More studies showed that tumor growth was promoted in the presence of MSCs in the TME, thus indicating that MSCs promote resistance to therapy, which is included among hematological malignancies. For example, in chronic myeloid leukemia (CML), MSCs protected CML cells from imatinib-induced cell death via the CXCL12/CXCR4 axis, which decreased caspase 3 activity [87]. Similarly, in chronic lymphoid leukemia (CLL), forodesine-induced CLL cell apoptosis was antagonized by MSCs, which inhibited forodesine-induced RNA and protein synthesis activity and increased the expression of the antiapoptotic protein Mcl-1 at both the transcript and protein levels [88]. Moreover, in multiple myeloma (MM), it was found that MSCs enhanced MM cell resistance to bortezomib through CXCL13 production [89]. In solid tumors, MSCs have also been shown to promote resistance to therapeutic therapy. For instance, in head and neck carcinoma, MSCs were reported to increase chemoresistance to paclitaxel by secreting several mediators, such as IL-7, IL-8, IGF and EGF [90]. In ovarian cancer, tumor-related MSCs were able to induce resistance to hyperthermic intraperitoneal chemotherapy by activating the CXCL12-CXCR4 axis, and when the CXCL12/CXCR4 interaction was blocked, the cytotoxicity of hyperthermia was restored [91]. In addition, the expression of IL-6 produced by MSCs is another key mechanism of resistance. IL- 6 raised the levels of Bcl-2 and $\mathrm{Bcl}-\mathrm{X}_{\mathrm{L}}$, which inhibited cancer cell apoptosis after cytotoxic therapy [92]. Moreover, MSC-secreted IL-6 has been shown to enhance tumor resistance by increasing the formation of CSCs [57]. In colorectal cancer, tumor cells release IL-1, which induces MSCs to secrete $\mathrm{PGE}_{2}$ and raises IL-6 level, which leads to the development of CSCs [93]. In addition to paracrine mechanism, the process of systematical secretion by MSCs is also implicated in the promotion of therapeutic resistance. Recently, Roodhart et al. showed that endogenous MSCs exposed to cisplatin became activated to protect tumor cells against chemotherapeutics through systematically secreting two different platinum-induced polyunsaturated fatty acids (PIFAs), 12-oxo-5,8,10-heptadecatrienoic acid (KHT) and hexadeca-4,7,10,13-tetraenoic acid [16:4(n3)]. Both PIFAs induced resistance to a range of chemotherapeutic agents in minute quantities. Moreover, the author discovered that blocking key enzymes implicated in PIFA synthesis (such as cyclooxygenase-1 and thromboxane synthase) inhibited MSC-induced resistance [94]. Their findings revealed that MSCs may generate chemoresistance even when they are not from tumors, and that MSCs can function as strong mediators of therapeutic resistance (Fig. 1).

In addition to secreting mediators, MSCs have the ability to transform into CSCs or provide CSC niche support. CSCs are a type of cancer cells that reside in tumor tissues at a low level, and they are resistant to many cytotoxic agents, partly because of their low proliferative rate, high level of membranal transporters that could control cytotoxic drug influx, and high DNA repair ability [95, 96]. Here, we will discuss how MSCs contribute to therapeutic resistance through this mechanism. Several tumor suppressor genes in MSCs, such as ras-associated family protein isoforms 1A (RassF1A) and hypermethylated in cancer 1 (HIC1), were methylated, which causes MSCs to convert into CSC cells and demonstrates MSCs' tumorigenic potential. In vitro, MSCs which were targeted methylations of HIC1/RassF1A lost anchorage dependence, and increased drug resistance, colony formation capability and pluripotency. In animal model, minimal quantities of targeted MSCs were injected in immunodeficient mice, resulting in tumor formation. These findings suggested that MSCs might convert into CSCs, increasing chemoresistance and allowing tumor recurrence after treatment cessation [97]. Moreover, MSCs were indicated to regulate CSCs to facilitate tumor growth and chemoresistance. In pancreatic ductal adenocarcinoma (PDAC), MSCs exposed to gemcitabine significantly enhanced CXCL10 expression, which further promoted CSC proliferation by overexpressing the CXCL10 receptor (CXCR3) on CSCs. As a result, tumor growth and chemoresistance were both boosted [98] (Fig. 1). Furthermore, MSCs pretreated with cisplatin altered the phosphorylation state of several tyrosine kinases, such as WNK1, c-Jun, STAT3 and $\mathrm{p} 53$, and produced factors that turn on the changes in stemness and resistance of tumor cells, thus promoting therapeutic resistance of tumor cells [99] (Table 2). However, it is not clear how MSCs and these mediators contribute to therapeutic resistance.

\section{MSCs inhibit therapeutic resistance}

In addition to promoting therapeutic resistance, MSCs were reported to increase sensitivity to therapies. The presence of CSCs in tumor tissues is the most common cause of therapeutic resistance and disease relapse [100], and STAT3 contributes to the maintenance of CSCs programs in cancer cells [101], so He and colleagues examined the expression of several stemness genes related to CSCs (Sox2, Oct4, Nanog, and c-Myc) as well as STAT3 signaling-related genes ( $\mathrm{p} 53$, cyclin D1, Bcl- $\mathrm{X}_{\mathrm{L}}$ ) in MDAMB-231 cells. Their results showed that the levels of these genes were significantly reduced in the MSCs-CM and the combination groups (radiation combined with MSC therapy). Thus, they concluded that MSCs could 


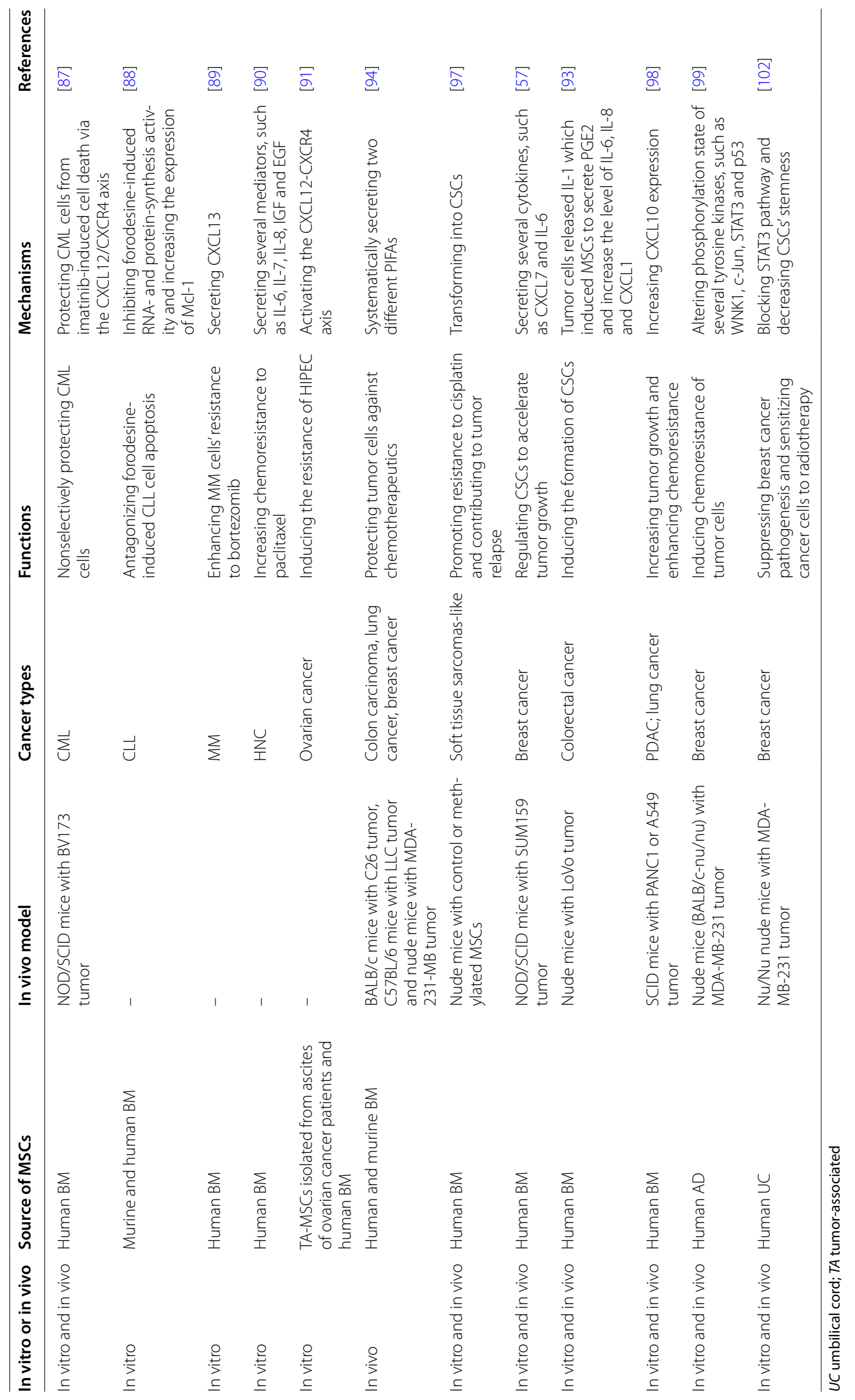


suppress breast cancer pathogenesis and sensitize cancer cells to radiotherapy through down-regulating the STAT3 pathway, providing a novel therapeutic for breast cancer in terms of overcoming radioresistance [102] (Table 2) (Fig. 1). In addition, engineered-MSCs were able to inhibit therapeutic resistance (Table 3). However, because the number of relevant studies is limited, additional researches are needed to fully understand the role of MSCs in therapeutic resistance.

\section{MSCs: a double-edged sword}

MSCs were reported to have pro- and anti-cancer effects. The opposing effects may depend on several factors. For example, the roles of MSCs in cancer progression differ depending on the tumor model employed. In an in vivo model of KS, bone marrow derived MSCs (BMSCs) administered intravenously (i.v.) home to carcinogenesis sites and potently suppress tumor growth. And in vitro, co-culture of MSCs with KS cells inhibit the proliferation of cancer cells. This effect of inhibiting tumor growth requires the BMSCs to achieve direct cell-cell contact via blocking the activation of Akt signaling [77]. In contrast, in an in vivo model of osteosarcoma, BMSCs injected i.v. targeted the osteosarcoma site and encouraged its growth and pulmonary metastasis, and in vitro, osteosarcoma cells' proliferation was enhanced in the presence of BMSC-CM, suggesting a contact-independent mechanism [103]. Moreover, the differentiation degree and source of MSCs also affect MSCs' functions. For example, adipocyte-differentiated MSCs significantly reduced all-trans retinoic acid- and doxorubicin-induced apoptosis of acute promyelocytic leukemia cells in vitro. MSCs' protective properties were attributed to the synthesis of leptin by adipocyte-differentiated MSCs. This antiapoptotic action of leptin required the stimulation of STAT3 and MAPK signaling [104]. Similarly, osteoblasts

Table 3 Antitumor molecules delivered by MSCs

\begin{tabular}{|c|c|c|c|}
\hline Molecules & Functions & Cancer types & References \\
\hline$\| \mathrm{L}-2$ & $\begin{array}{l}\text { Delaying tumor growth and developing CD8-mediated tumor- } \\
\text { specific anticancer immunity }\end{array}$ & Melanoma & {$[114]$} \\
\hline IL-12 & $\begin{array}{l}\text { Boosting antitumor T cell responses and inhibiting tumor } \\
\text { growth }\end{array}$ & Melanoma; cervical cancer & {$[113]$} \\
\hline IL-18 & Activating T cells & GBM & {$[143]$} \\
\hline NK4 & Inhibiting angiogenesis and promoting apoptosis & Lung cancer & {$[144]$} \\
\hline IFN- $\beta$ & Inhibiting the growth of cancer cells & Melanoma; metastatic prostate cancer & {$[115,145]$} \\
\hline IFN- $\gamma$ & Immunostimulation and stimulating apoptosis & Leukemia & {$[146]$} \\
\hline $\mathrm{CX} 3 \mathrm{CL}_{1}$ & Activating $\mathrm{CD}^{+}$and $\mathrm{NK}$ cells & Lung cancer & {$[147]$} \\
\hline PE-cytotoxins & - & GBM & {$[148]$} \\
\hline sFlt-1 & Inhibiting angiogenesis and metastasis & Tumor lung metastasis & {$[149]$} \\
\hline ¡NOS & Inhibiting growth of tumor cells & Fibrosarcoma & {$[150]$} \\
\hline HSV-TK & Transferring ganciclovir into active cytotoxic drugs & GBM & {$[112]$} \\
\hline Nanoparticle & Drug-loaded polymeric nanoparticles & Lung cancer & {$[83]$} \\
\hline TRAIL & Inducing apoptosis & Lung cancer & {$[82]$} \\
\hline Cytosine deaminase & $\begin{array}{l}\text { Converting inactive systemically administered prodrugs into } \\
\text { active cytotoxic agents }\end{array}$ & Colon cancer; prostate cancer & {$[110,111]$} \\
\hline rCE & Converting the CPT-11 to SN-38 & GBM & {$[151]$} \\
\hline miR-124a & Decreasing survival of cancer cells & GBM & {$[152]$} \\
\hline miR-143 & Inhibiting cancer migration & Osteosarcoma & {$[153]$} \\
\hline miR-146b & Inhibiting cancer cells growth & GBM & {$[154]$} \\
\hline Cytotoxic chemotherapy agents & Inhibiting cell viability and reducing cancer cell growth & Oral squamous cancer & {$[128]$} \\
\hline miR-193a & $\begin{array}{l}\text { Reducing cisplatin resistance of NSCLC cells by downregulat- } \\
\text { ing LRRC } 1\end{array}$ & NSCLC & {$[131]$} \\
\hline anti-miR-9 & $\begin{array}{l}\text { Reversing the chemoresistance of GBM cells to TMZ by regulat- } \\
\text { ing the expression of multidrug transporter }\end{array}$ & GBM & {$[132]$} \\
\hline siGRP78 & Reversing the drug resistance & $\mathrm{HCC}$ & {$[133]$} \\
\hline miR-122 & $\begin{array}{l}\text { Enhancing cancer cells chemosensitivity to sorafenib by down- } \\
\text { regulating the expression of CCNG1, ADAM10 and IGF1R }\end{array}$ & $\mathrm{HCC}$ & {$[134]$} \\
\hline MiR-6785-5p & Suppressing angiogenesis and metastasis in GC & GC & [155] \\
\hline
\end{tabular}

NK4 HGF antagonist/angiogenesis inhibitor; PE-cytotoxins pseudomonas exotoxin-cytotoxins; sFlt-1 soluble fms-like tyrosine kinase 1; iNOS inducible nitric oxide synthase; $r C E$ rabbit carboxylesterase; NK cells natural killer cells; GC gastric cancer 
differentiated from MSCs increased acute myelogenous leukemia (AML) cell engraftment of in BM and protected AML cells from chemotherapy-induced death [105]. On the other hand, MSCs derived from human umbilical cord blood and adipose tissue induced apoptosis of breast cancer cells by enhancing PARP and caspase- 3 cleavage [106]. In addition, it was revealed that MSC functions differ in vitro and in vivo, and this difference has been demonstrated in various cancer cell lines. MSCs showed an antiproliferative role by causing G1 arrest in vitro; however, mice injected with cancer cells and MSCs exhibited faster tumor growth in vivo [107]. These results exposed a significant roadblock in the process of putting anticancer MSCs into clinical therapy. Despite the fact that numerous studies have demonstrated that MSCs have a protumorigenic effect, they were genetically engineered as a vehicle to target cancer cells. This method has been proven to be effective in multiple cancer models [82-84] (Table 3). In summary, MSCs are a group of pluripotent cells that may migrate to tumor sites and have a positive or negative impact in cancer progression via different mechanisms.

\section{MSCs as a potential therapeutic avenue for cancer}

MSCs' immunosuppressive and regenerative abilities suggest that they might be used to treat a variety of illnesses. Meanwhile, MSCs have been chosen as drug delivery vehicles in a variety of cancers because of their propensity to homing to tumor locations. For example, MSCs were used to deliver oncolytic viral loads into tumors, thereby effectively killing cancer cells [108, 109]. Moreover, MSCs were genetically engineered to express specific enzymes, such as herpes simplex virus-thymidine kinase (HSV-TK) and cytosine deaminase, which have the ability to convert inactive systemically administered prodrugs into active cytotoxic agents, thus further enhancing chemotherapy sensitivity and reducing potential toxicity [110-112]. These data suggested that MSCs were efficient anticancer delivery agents, improving tumor killing specificity and decreasing systemic toxicity.

Another method for designing MSCs is that MSCs continuously generate unique immunomodulatory cytokines that can induce cancer cell death. For example, MSCs with IL-12 overexpression enhanced antitumor T cell responses and inhibited tumor growth [113]. In melanoma, IL-2-expressing MSCs were indicated to delay tumor growth, developing CD8-mediated tumorspecific anticancer immunity [114]. In melanoma and metastatic prostate cancer, IFN $\beta$-expressing MSCs inhibited the growth of cancer cells [115]. Moreover, MSCs with genetic alterations can directly target cancer cells. For instance, TRAIL-expressing MSCs have been shown to effectively kill cancer cells in multiple cancer models, such as lung, glioblastoma (GBM), pancreatic and colorectal cancers [116-120]. In addition, TRAIL-expressing MSCs can directly target CSCs in lung cancer, reducing tumor aggressiveness and chemoresistance as well as relapse [121]. Furthermore, MSCs were also utilized to treat residual disease that has undergone chemotherapy, radiation, or surgery. In GBM, MSCs engineered with TRAIL and/or oncolytic viruses were able to effectively kill residual tumor cells [122, 123]. More data have been included in Table 3. Taken together, genetically modified MSCs appear to be a potential cancer treatment option.

However, MSC-based cell treatment may have several potential drawbacks, including noneffective local concentrations of drugs within tumors and nonspecific dissemination throughout the tissue and organism [124]. In addition, their physiological function of differentiating into mesenchymal lineages may enhance immunogenicity, lower therapeutic potential, and promote tumorigenesis [125]. To overcome these obstacles, extracellular vehicles (EVs) generated from MSCs have been disclosed as a drug delivery strategy for killing cancer cells. Similar to parental MSCs, such EVs still have tumor homing ability [126] and immune-suppressive properties [127]. Emerging evidence indicated that EVs were designed to overexpress cytotoxic chemotherapy agents, selectively killing cancer cells. For example, in oral squamous cancer, paclitaxel-, doxorubicin- or gemcitabine-expressing EVs inhibited cell viability and reduced cancer cell growth [128] (Table 3). Moreover, in the same cancer type, MSCs are also implicated in the composition of $\mathrm{M} / \mathrm{LPV} / \mathrm{O}_{2}$, which increases targeting efficacy and overcomes tumor hypoxia-associated resistance in sonodynamic therapy, thus enhancing therapeutic outcomes [129]. In addition, Exo, a kind of EVs, has been linked to cancer progression. Exo are enclosed vesicles with small membranes that communicate with other cells. They can load several molecules, including lipids, proteins, nucleic acids, mRNA and miRNA [130]. For example, in non-small cell lung cancer (NSCLC), Wu et al. found that exosomal miR193a in MSCs reduces the cisplatin resistance of NSCLC cells by downregulating leucine-rich repeat-containing protein 1 (LRRC1), thus providing new insights into a novel therapeutic for NSCLC [131]. In contrast to Wu's work, Jessian and colleagues performed anti-miR delivery by MSC-derived Exos to explore the chemosensitivity of GBM cells. Their findings demonstrated that the delivery of MSC-Exo-anti-miR-9 to cancer cells reversed the chemoresistance of GBM cells to temozolomide by regulating the expression of multidrug transporters. Their report revealed a particular target for GBM via anti-miR delivery in the RNA therapeutics field [132]. Similarly, in hepatocellular carcinoma (HCC), Li et al. manipulated MSCs to express exosomal siGRP78, which can target 
GRP78 overexpressed in resistant HCC cells. Their findings suggested that siGRP78-modified MSC-Exos might sensitize sorafenib-resistant HCC cells to sorafenib to reverse drug resistance [133]. Additionally, in HCC, Lou et al. indicated that MSCs Exos promoted miR-122-enhanced HCC cell chemosensitivity to sorafenib in HCC cells by downregulating the expression of miR-122 target genes, such as cyclin G1 (CCNG1), disintegrin and metalloprotease 10 (ADAM10), and insulin-like growth factor receptor 1 (IGF1R), which are involved in the drug sensitivity or resistance of cancer cells. Their study supported that MSC exosome-miR-122 is a novel strategy to increase HCC chemosensitivity [134] (Table 3). In PDAC, paclitaxel (PTX) and gemcitabine monophosphate (GEMP) were loaded in/on BMSC Exos in Zhou's study. The "Exo" platform they constructed was capable of overcoming chemoresistance because it had a tumorhoming property to overstep the barriers of pathological extracellular matrix to further enhance the accumulation of PTX and GEMP in the tumor site [135]. Based on combined studies, the MSC delivery system may serve as a promising strategy for inhibiting therapeutic resistance in cancer treatment.

Nevertheless, long-term cultures of MSCs for treatment were shown to commonly undergo spontaneous malignant transformation, with transformed mesenchymal cells leading to tumors in vivo [136]. Then after 5 years, the initial report was retracted because the authors were unable to replicate some of the reported spontaneous transformation events and suspected that the phenomenon is due to a cross-contamination artifact [137]. These findings highlight the need of following strict cell culture methods when it comes to medicinal reasons. Moreover, genetic abnormalities were also observed in vitro [138]. Notably, there are no clear evidence that chromosomal changes lead to malignant transformation in vitro or in vivo [139]. In addition, an examination of tissues following MSC treatment in humans revealed no evidence of malignant tumors originating from MSCs [140]. Even yet, we can't rule out the possibility of malignancies forming after MSC therapy since chromosomal abnormalities in MSCs may occur at the time of injection or afterward. More patient follow-up research on MSC therapy should be required. Out of an abundance of caution, standardized purification and expansion protocols must be created, given the possibility of chromosomal aberrations under culture conditions [141]. As a result, culture conditions with low proliferation rates and limited expansion rates have been proposed to reduce the likelihood of acquired chromosomal abnormalities [141].

Generally, the combined treatments of MSCs and other existing treatment modalities have proven to be a promising therapeutic in several cancer types, and simultaneously, uniform purification and expansion protocols in vitro must be followed.

\section{Conclusions}

The roles of MSCs in cancer progression and anticancer therapeutic resistance are of versatility and plasticity. MSCs have been implicated in promoting cancer, including promoting the survival, metastasis, angiogenesis and evasion of the immune system, as well as inhibiting apoptosis. MSCs facilitate cancer progression and therapeutic resistance by close interactions with cancer cells or systemic/paracrine mechanisms involving secreted factors. MSC-targeting treatment might represent an anticancer therapy and improve therapeutic sensitivity. MSCs show diverse mechanisms of enhancing cancer progression in vitro; however, whether MSCs play the same roles in vivo has been undetermined. As a result, inhibiting MSCs in a clinical setting becomes a challenge. The ability of MSCs homing to tumor sites aids in the precise targeting of malignancies. However, it is worth noting that these genetically engineered MSCs may have inherent mechanisms that facilitate cancer cell proliferation, metastasis and therapeutic resistance, implying that engineered MSCs used to target tumors might have unintended consequences if handled inappropriately. Although numerous studies have shown that MSCs have cancer-promoting properties, several publications have shown that MSCs can also inhibit cancer progression. Different stimuli on MSCs depend on the MSC status and have positive or negative effects on cancer etiology. However, the precise mechanisms of these phenomena are still not clear. In summary, MSCs have key effects on tumor growth and treatment response; thus, MSCs alone or in combination with other treatments may become a promising treatment for cancer. To address the clinical challenges outlined above, additional researches should be required.

\section{Abbreviation}

MSCs: Mesenchymal stem cells; BM: Bone marrow; TME: Tumor microenvironment; TLR: Toll-like receptors; PGE $_{2}$ : Prostaglandin $E_{2} ;$ IDO: Indoleamine 2,3-dioxygenase; GARP: Glycoprotein a repetitions predominant; Treg: Regulatory T cells; CM: Conditioned medium; FAO: Fatty acid oxidation; KS: Kaposi's sarcoma; DUSP1: Dual-specificity phosphatase 1; TRAIL: TNF-related apoptosisinducing ligand; BMP4: Bone morphogenetic protein 4; CML: Chronic myeloid leukemia; CLL: Chronic lymphoid leukemia; MM: Multiple myeloma; PIFAs: Platinum-induced polyunsaturated fatty acids; CSCs: Cancer stem cells; RassF1A: Ras-associated family protein isoforms $1 \mathrm{~A} ; \mathrm{HIC}$ : Hypermethylated in cancer 1 ; PDAC: Pancreatic ductal adenocarcinoma; BMSCs: Bone marrow derived MSCs; AML: Acute myelogenous leukemia; GBM: Glioblastoma multiforme; EVs:

Extracellular vehicles; SDT: Sonodynamic therapy; NSCLC: Non-small cell lung cancer; LRRC1: Leucine-rich repeat-containing protein 1; HCC: Hepatocellular carcinoma; PTX: Paclitaxel; GEMP: Gemcitabine monophosphate; CAF: Cancerassociated fibroblasts; EMT: Epithelial-mesenchymal transition. 


\section{Acknowledgements}

All authors approved this paper. We also thank the contributions of the additional new 5 other authors, Juanmei Cao, Fangqi Chen, Ting Wu, Siyuan Chen and Li Zhu, including editing English grammar and polishing language.

\section{Authors' contributions}

XYX and CXT drafted the manuscript. MJZ, YHS and CZH edited the work. All authors read and approved the final manuscript.

\section{Funding}

This work was supported by Grants from the General Program of the National Natural Science Foundation of China (No. 81972565).

\section{Availability of data and materials}

Not applicable.

\section{Declarations}

Ethics approval and consent to participate

Not applicable.

\section{Consent for publication}

Not applicable.

\section{Competing interests}

The authors declare no competing interests.

\section{Author details}

${ }^{1}$ Department of Dermatology, Union Hospital, Tongji Medical College, Huazhong University of Science and Technology, Wuhan 430022, Hubei, China. ${ }^{2}$ Department of Cardiology, Hubei Provincial Hospital of TCM, Wuhan 430022, Hubei, China. ${ }^{3}$ Department of Dermatology, Zhongnan Hospital, Wuhan University, Wuhan 430022, Hubei, China. ${ }^{4}$ Department of Dermatology, Second Affiliated Hospital, Zhejiang University School of Medicine, Hangzhou 310009, Zhejiang, China.

Received: 19 June 2021 Accepted: 26 October 2021

Published online: 04 November 2021

\section{References}

1. Li N, Hua J. Interactions between mesenchymal stem cells and the immune system. Cell Mol Life Sci. 2017:74(13):2345-60.

2. Wexler SA, et al. Adult bone marrow is a rich source of human mesenchymal 'stem' cells but umbilical cord and mobilized adult blood are not. Br J Haematol. 2003;121(2):368-74.

3. Dazzi $F$, et al. The role of mesenchymal stem cells in haemopoiesis. Blood Rev. 2006;20(3):161-71.

4. Miana VV, Gonzalez EAP. Adipose tissue stem cells in regenerative medicine. Ecancermedicalscience. 2018;12:822.

5. Zhang $L$, et al. Therapeutic effect of human umbilical cord-derived mesenchymal stem cells on injured rat endometrium during its chronic phase. Stem Cell Res Ther. 2018;9(1):36.

6. Park EH, et al. Intravenous infusion of umbilical cord blood-derived mesenchymal stem cells in rheumatoid arthritis: a phase in clinical trial. Stem Cells Transl Med. 2018;7(9):636-42.

7. Li JY, et al. Human amniotic mesenchymal stem cells and their paracrine factors promote wound healing by inhibiting heat stress-induced skin cell apoptosis and enhancing their proliferation through activating PI3K/AKT signaling pathway. Stem Cell Res Ther. 2019;10(1):247.

8. Bier A, et al. Placenta-derived mesenchymal stromal cells and their exosomes exert therapeutic effects in Duchenne muscular dystrophy. Biomaterials. 2018;174:67-78.

9. Longhini ALF, et al. Peripheral blood-derived mesenchymal stem cells demonstrate immunomodulatory potential for therapeutic use in horses. PLoS ONE. 2019;14(3):e0212642.
10. Jackson WM, Nesti LJ, Tuan RS. Potential therapeutic applications of muscle-derived mesenchymal stem and progenitor cells. Expert Opin Biol Ther. 2010;10(4):505-17.

11. Cheng T, et al. Lung-resident mesenchymal stem cells regulated the inflammatory responses in innate and adaptive immune cells through HVEM-BTLA pathway during ARDS. Exp Cell Res. 2020;395(1):112155.

12. Leuning DG, et al. The cytokine secretion profile of mesenchymal stromal cells is determined by surface structure of the microenvironment. Sci Rep. 2018:8(1):7716.

13. Werts ED, et al. Characterization of marrow stromal (fibroblastoid) cells and their association with erythropoiesis. Exp Hematol. 1980;8(4):423-33.

14. Kuznetsov SA, et al. Single-colony derived strains of human marrow stromal fibroblasts form bone after transplantation in vivo. J Bone Miner Res. 1997;12(9):1335-47.

15. Colter DC, Sekiya I, Prockop DJ. Identification of a subpopulation of rapidly self-renewing and multipotential adult stem cells in colonies of human marrow stromal cells. Proc Natl Acad Sci USA. 2001;98(14):7841-5.

16. Fu X, et al. Mesenchymal stem cell migration and tissue repair. Cells. 2019;8(8):784

17. Reagan MR, Kaplan DL. Concise review: mesenchymal stem cell tumorhoming: detection methods in disease model systems. Stem Cells. 2011;29(6):920-7.

18. Chen X, Armstrong MA, Li G. Mesenchymal stem cells in immunoregulation. Immunol Cell Biol. 2006;84(5):413-21.

19. Zhao $\mathrm{L}$, et al. The role of mesenchymal stem cells in hematopoietic stem cell transplantation: prevention and treatment of graft-versushost disease. Stem Cell Res Ther. 2019:10(1):182.

20. Yuan X, et al. Mesenchymal stem cell therapy induces FLT3L and CD1c(+) dendritic cells in systemic lupus erythematosus patients. Nat Commun. 2019;10(1):2498.

21. De Becker A, Riet IV. Homing and migration of mesenchymal stromal cells: how to improve the efficacy of cell therapy? World J Stem Cells. 2016;8(3):73-87.

22. Ponte $\mathrm{AL}$, et al. The in vitro migration capacity of human bone marrow mesenchymal stem cells: comparison of chemokine and growth factor chemotactic activities. Stem Cells. 2007:25(7):1737-45.

23. Schmidt $A$, et al. Basic fibroblast growth factor controls migration in human mesenchymal stem cells. Stem Cells. 2006;24(7):1750-8.

24. Li L, et al. HIF-1alpha promotes a hypoxia-independent cell migration. Open Biol J. 2010:3:8-14.

25. Dwyer RM, et al. Monocyte chemotactic protein-1 secreted by primary breast tumors stimulates migration of mesenchymal stem cells. Clin Cancer Res. 2007;13(17):5020-7.

26. Abarbanell AM, et al. Proinflammatory cytokine effects on mesenchymal stem cell therapy for the ischemic heart. Ann Thorac Surg 2009;88(3):1036-43.

27. Chen MS, et al. IL-1 beta-induced matrix metalloprotease-1 promotes mesenchymal stem cell migration via PAR1 and G-protein-coupled signaling pathway. Stem Cells Int. 2018:2018:3524759.

28. Dubon MJ, et al. Transforming growth factor beta induces bone marrow mesenchymal stem cell migration via noncanonical signals and N-cadherin. J Cell Physiol. 2018;233(1):201-13.

29. Lourenco $S$, et al. Macrophage migration inhibitory factor-CXCR4 is the dominant chemotactic axis in human mesenchymal stem cell recruitment to tumors. J Immunol. 2015;194(7):3463-74.

30. Ba L, et al. Gastric cancer cell-derived exosomes can regulate the biological functions of mesenchymal stem cells by inducing the expression of circular RNA circ 0004303. Stem Cells Dev. 2021:30(16):830-42.

31. Schlosser $\mathrm{S}$, et al. Paracrine effects of mesenchymal stem cells enhance vascular regeneration in ischemic murine skin. Microvasc Res. 2012;83(3):267-75.

32. Shome S, Dasgupta PS, Basu S. Dopamine regulates mobilization of mesenchymal stem cells during wound angiogenesis. PLOS ONE. 2012;7(2):e31682

33. Nilendu P, et al. Mutual concessions and compromises between stroma cells and cancer cells: driving tumor development and drug resistance. Cell Oncol. 2018:41(4):353-67.

34. Spaw M, Anant S, Thomas SM. Stromal contributions to the carcinogenic process. Mol Carcinog. 2017:56(4):1199-213. 
35. Waterman RS, et al. A new mesenchymal stem cell (MSC) paradigm: polarization into a pro-inflammatory MSC1 or an Immunosuppressive MSC2 phenotype. PLOS ONE. 2010;5(4):e10088.

36. Waterman RS, Henkle SL, Betancourt AM. Mesenchymal stem cell 1 (MSC1)-based therapy attenuates tumor growth whereas MSC2treatment promotes tumor growth and metastasis. PLOS ONE. 2012;7(9):e45590.

37. Rivera-Cruz CM, et al. The immunomodulatory effects of mesenchymal stem cell polarization within the tumor microenvironment niche. Stem Cells Int. 2017;2017:4015039

38. Nemeth $\mathrm{K}$, et al. Bone marrow stromal cells attenuate sepsis via prostaglandin $\mathrm{E}(2)$-dependent reprogramming of host macrophages to increase their interleukin-10 production. Nat Med. 2009;15(1):42-9.

39. Bai $L$, et al. Human bone marrow-derived mesenchymal stem cells induce Th2-polarized immune response and promote endogenous repair in animal models of multiple sclerosis. Glia. 2009;57(11):1192-203.

40. Niu J, et al. Mesenchymal stem cells inhibit T cell activation by releasing TGF-beta1 from TGF-beta1/GARP complex. Oncotarget. 2017;8(59):99784-800

41. Meisel R, et al. Human bone marrow stromal cells inhibit allogeneic T-cell responses by indoleamine 2,3-dioxygenase-mediated tryptophan degradation. Blood. 2004;103(12):4619-21.

42. Fallarino F, et al. The combined effects of tryptophan starvation and tryptophan catabolites down-regulate $T$ cell receptor zeta-chain and induce a regulatory phenotype in naive T cells. J Immunol. 2006:176(11):6752-61.

43. Lee $\mathrm{HJ}$, et al. Mesenchymal stem/stromal cells protect against autoimmunity via CCL2-dependent recruitment of myeloid-derived suppressor cells. J Immunol. 2015;194(8):3634-45.

44. Asari $\mathrm{S}$, et al. Mesenchymal stem cells suppress B-cell terminal differentiation. Exp Hematol. 2009;37(5):604-15.

45. Ungerer C, et al. Galectin-9 is a suppressor of T and B cells and predicts the immune modulatory potential of mesenchymal stromal cell preparations. Stem Cells Dev. 2014:23(7):755-66

46. Galland S, et al. Tumor-derived mesenchymal stem cells use distinct mechanisms to block the activity of natural killer cell subsets. Cell Rep. 2017;20(12):2891-905.

47. Spaggiari GM, et al. MSCs inhibit monocyte-derived DC maturation and function by selectively interfering with the generation of immature DCs: central role of MSC-derived prostaglandin E2. Blood. 2009:113(26):6576-83.

48. Jiang XX, et al. Human mesenchymal stem cells inhibit differentiation and function of monocyte-derived dendritic cells. Blood. 2005;105(10):4120-6.

49. Chen B, et al. Bone marrow-derived mesenchymal stem cells exert diverse effects on different macrophage subsets. Stem Cells Int. 2018;2018:8348121.

50. Vasandan $A B$, et al. Human Mesenchymal stem cells program macrophage plasticity by altering their metabolic status via a PGE2dependent mechanism. Sci Rep. 2016;6:38308.

51. Hu X, et al. Programming of the development of tumor-promoting neutrophils by mesenchymal stromal cells. Cell Physiol Biochem. 2014;33(6):1802-14

52. Zhu Q, et al. The IL-6-STAT3 axis mediates a reciprocal crosstalk between cancer-derived mesenchymal stem cells and neutrophils to synergistically prompt gastric cancer progression. Cell Death Dis. 2014;5:e1295.

53. Zhang T, et al. Bone marrow-derived mesenchymal stem cells promote growth and angiogenesis of breast and prostate tumors. Stem Cell Res Ther. 2013;4(3):70

54. Li GC, et al. Mesenchymal stem cells promote tumor angiogenesis via the action of transforming growth factor beta1. Oncol Lett. 2016;11(2):1089-94.

55. Yuan Z, et al. LncRNA H19 knockdown in human amniotic mesenchymal stem cells suppresses angiogenesis by associating with EZH2 and activating vasohibin-1. Stem Cells Dev. 2019:28(12):781-90.

56. Martin FT, et al. Potential role of mesenchymal stem cells (MSCs) in the breast tumour microenvironment: stimulation of epithelial to mesenchymal transition (EMT). Breast Cancer Res Treat. 2010;124(2):317-26.

57. Liu S, et al. Breast cancer stem cells are regulated by mesenchymal stem cells through cytokine networks. Cancer Res. 2011;71(2):614-24.
58. Kaplan RN, et al. VEGFR1-positive haematopoietic bone marrow progenitors initiate the pre-metastatic niche. Nature. 2005:438(7069):820-7

59. Bergfeld SA, DeClerck YA. Bone marrow-derived mesenchymal stem cells and the tumor microenvironment. Cancer Metastasis Rev. 2010;29(2):249-61.

60. Karnoub AE, et al. Mesenchymal stem cells within tumour stroma promote breast cancer metastasis. Nature. 2007;449(7162):557-63.

61. Hung SC, et al. Angiogenic effects of human multipotent stromal cell conditioned medium activate the PI3K-Akt pathway in hypoxic endothelial cells to inhibit apoptosis, increase survival, and stimulate angiogenesis. Stem Cells. 2007;25(9):2363-70.

62. Dias $\mathrm{S}$, et al. Vascular endothelial growth factor (VEGF)-C signaling through FLT-4 (VEGFR-3) mediates leukemic cell proliferation, survival, and resistance to chemotherapy. Blood. 2002;99(6):2179-84.

63. Konig A, et al. Basic fibroblast growth factor (bFGF) upregulates the expression of bcl-2 in B cell chronic lymphocytic leukemia cell lines resulting in delaying apoptosis. Leukemia. 1997;11(2):258-65.

64. Brogi $E$, et al. Indirect angiogenic cytokines upregulate VEGF and bFGF gene expression in vascular smooth muscle cells, whereas hypoxia upregulates VEGF expression only. Circulation. 1994;90(2):649-52.

65. Burger JA, et al. Blood-derived nurse-like cells protect chronic lymphocytic leukemia B cells from spontaneous apoptosis through stromal cell-derived factor-1. Blood. 2000;96(8):2655-63.

66. Efimenko A, et al. Angiogenic properties of aged adipose derived mesenchymal stem cells after hypoxic conditioning. J Transl Med. 2011;9:10.

67. Stamler JS. Redox signaling: nitrosylation and related target interactions of nitric oxide. Cell. 1994;78(6):931-6.

68. Naderi EH, et al. Bone marrow stroma-derived PGE2 protects BCP-ALL cells from DNA damage-induced p53 accumulation and cell death. Mol Cancer. 2015:14:14.

69. Bonuccelli $\mathrm{G}$, et al. Role of mesenchymal stem cells in osteosarcoma and metabolic reprogramming of tumor cells. Oncotarget. 2014;5(17):7575-88.

70. Miyazaki Y, et al. Adipose-derived mesenchymal stem cells differentiate into pancreatic cancer-associated fibroblasts in vitro. FEBS Open Bio. 2020;10(11):2268-81.

71. Liu B, et al. Aberrant mannosylation profile and FTX/miR-342/ALG3axis contribute to development of drug resistance in acute myeloid leukemia. Cell Death Dis. 2018;9(6):688.

72. Yuan SX, et al. Long noncoding RNA DANCR increases stemness features of hepatocellular carcinoma by derepression of CTNNB1. Hepatology. 2016;63(2):499-511.

73. Wang Y, et al. The long noncoding RNA IncTCF7 promotes self-renewal of human liver cancer stem cells through activation of Wnt signaling. Cell Stem Cell. 2015;16(4):413-25.

74. He W, et al. MSC-regulated IncRNA MACC1-AS1 promotes stemness and chemoresistance through fatty acid oxidation in gastric cancer. Oncogene. 2019;38(23):4637-54.

75. Tu Z, et al. Microenvironmental regulation of long noncoding RNA LINC01133 promotes cancer stem cell-like phenotypic traits in triplenegative breast cancers. Stem Cells. 2019;37(10):1281-92.

76. Ohlsson LB, et al. Mesenchymal progenitor cell-mediated inhibition of tumor growth in vivo and in vitro in gelatin matrix. Exp Mol Pathol. 2003;75(3):248-55.

77. Khakoo AY, et al. Human mesenchymal stem cells exert potent antitumorigenic effects in a model of Kaposi's sarcoma. J Exp Med. 2006:203(5):1235-47.

78. Qiao L, et al. Dkk-1 secreted by mesenchymal stem cells inhibits growth of breast cancer cells via depression of Wnt signalling. Cancer Lett. 2008;269(1):67-77.

79. Lu YR, et al. The growth inhibitory effect of mesenchymal stem cells on tumor cells in vitro and in vivo. Cancer Biol Ther. 2008;7(2):245-51.

80. Otsu K, et al. Concentration-dependent inhibition of angiogenesis by mesenchymal stem cells. Blood. 2009;113(18):4197-205.

81. Gu H, et al. Mesenchymal stem cell-derived exosomes block malignant behaviors of hepatocellular carcinoma stem cells through a IncRNA C5orf66-AS1/microRNA-127-3p/DUSP1/ERK axis. Hum Cell. 2021;34(6):1812-29.

82. Loebinger MR, et al. TRAlL-expressing mesenchymal stem cells kill the putative cancer stem cell population. Br J Cancer. 2010;103(11):1692-7. 
83. Sadhukha T, O'Brien TD, Prabha S. Nano-engineered mesenchymal stem cells as targeted therapeutic carriers. J Control Release. 2014;196:243-51.

84. Li Q, et al. Mesenchymal stem cells from human fat engineered to secrete BMP4 are nononcogenic, suppress brain cancer, and prolong survival. Clin Cancer Res. 2014;20(9):2375-87.

85. Meads MB, Gatenby RA, Dalton WS. Environment-mediated drug resistance: a major contributor to minimal residual disease. Nat Rev Cancer. 2009:9(9):665-74.

86. Borst P. Cancer drug pan-resistance: pumps, cancer stem cells, quiescence, epithelial to mesenchymal transition, blocked cell death pathways, persisters or what? Open Biol. 2012;2(5):120066.

87. Vianello F, et al. Bone marrow mesenchymal stromal cells nonselectively protect chronic myeloid leukemia cells from imatinibinduced apoptosis via the CXCR4/CXCL12 axis. Haematologica. 2010;95(7):1081-9.

88. Balakrishnan $\mathrm{K}$, et al. Influence of bone marrow stromal microenvironment on forodesine-induced responses in CLL primary cells. Blood. 2010;116(7):1083-91.

89. Zhang G, et al. Mesenchymal stem cells from bone marrow regulate invasion and drug resistance of multiple myeloma cells by secreting chemokine CXCL13. Bosn J Basic Med Sci. 2020;20(2):209-17.

90. Scherzed A, et al. BMSC enhance the survival of paclitaxel treated squamous cell carcinoma cells in vitro. Cancer Biol Ther. 2011;11(3):349-57.

91. Lis R, et al. Tumor associated mesenchymal stem cells protects ovarian cancer cells from hyperthermia through CXCL12. Int J Cancer. 2011;128(3):715-25.

92. Dreuw A, et al. Interleukin-6-type cytokines upregulate expression of multidrug resistance-associated proteins in NHEK and dermal fibroblasts. J Invest Dermatol. 2005;124(1):28-37.

93. Li HJ, et al. Cancer-stimulated mesenchymal stem cells create a carcinoma stem cell niche via prostaglandin E2 signaling. Cancer Discov. 2012;2(9):840-55.

94. Roodhart JM, et al. Mesenchymal stem cells induce resistance to chemotherapy through the release of platinum-induced fatty acids. Cancer Cell. 2011;20(3):370-83.

95. Moitra K. Overcoming multidrug resistance in cancer stem cells. Biomed Res Int. 2015;2015:635745.

96. Steinbichler TB, et al. Therapy resistance mediated by cancer stem cells. Semin Cancer Biol. 2018:53:156-67.

97. Teng IW, et al. Targeted methylation of two tumor suppressor genes is sufficient to transform mesenchymal stem cells into cancer stem/initiating cells. Cancer Res. 2011;71(13):4653-63.

98. Timaner $M$, et al. Therapy-educated mesenchymal stem cells enrich for tumor-initiating cells. Cancer Res. 2018;78(5):1253-65.

99. Skolekova S, et al. Cisplatin-induced mesenchymal stromal cells-mediated mechanism contributing to decreased antitumor effect in breast cancer cells. Cell Commun Signal. 2016;14:4.

100. Al-Ejeh F, et al. Breast cancer stem cells: treatment resistance and therapeutic opportunities. Carcinogenesis. 2011;32(5):650-8

101. Fouse SD, Costello JF. Cancer stem cells activate STAT3 the EZ way. Cancer Cell. 2013;23(6):711-3.

102. He N, et al. MSCs inhibit tumor progression and enhance radiosensitivity of breast cancer cells by down-regulating Stat3 signaling pathway. Cell Death Dis. 2018:9(10):1026.

103. Xu WT, et al. Human mesenchymal stem cells (hMSCs) target osteosarcoma and promote its growth and pulmonary metastasis. Cancer Lett. 2009;281(1):32-41.

104. Tabe $Y$, et al. PML-RARalpha is associated with leptin-receptor induction: the role of mesenchymal stem cell-derived adipocytes in APL cell survival. Blood. 2004;103(5):1815-22.

105. Ishikawa F, et al. Chemotherapy-resistant human AML stem cells home to and engraft within the bone-marrow endosteal region. Nat Biotechnol. 2007;25(11):1315-21.

106. Sun B, et al. Therapeutic potential of mesenchymal stromal cells in a mouse breast cancer metastasis model. Cytotherapy. 2009;11(3):289-98.

107. Ramasamy $R$, et al. Mesenchymal stem cells inhibit proliferation and apoptosis of tumor cells: impact on in vivo tumor growth. Leukemia. 2007;21(2):304-10.
108. Mader EK, et al. Mesenchymal stem cell carriers protect oncolytic measles viruses from antibody neutralization in an orthotopic ovarian cancer therapy model. Clin Cancer Res. 2009;15(23):7246-55.

109. Kim J, et al. Stem cell-based cell carrier for targeted oncolytic virotherapy: translational opportunity and open questions. Viruses. 2015;7(12):6200-17.

110. Kucerova $L$, et al. Adipose tissue-derived human mesenchymal stem cells mediated prodrug cancer gene therapy. Cancer Res. 2007;67(13):6304-13.

111. Cavarretta IT, et al. Adipose tissue-derived mesenchymal stem cells expressing prodrug-converting enzyme inhibit human prostate tumor growth. Mol Ther. 2010;18(1):223-31.

112. Uchibori $R$, et al. Retroviral vector-producing mesenchymal stem cells for targeted suicide cancer gene therapy. J Gene Med. 2009;11(5):373-81

113. Seo SH, et al. The effects of mesenchymal stem cells injected via different routes on modified IL-12-mediated antitumor activity. Gene Ther. 2011;18(5):488-95.

114. Stagg J, et al. Marrow stromal cells for interleukin-2 delivery in cancer immunotherapy. Hum Gene Ther. 2004;15(6):597-608.

115. Studeny $M$, et al. Bone marrow-derived mesenchymal stem cells as vehicles for interferon-beta delivery into tumors. Cancer Res. 2002;62(13):3603-8.

116. Yu R, et al. Delivery of sTRAIL variants by MSCs in combination with cytotoxic drug treatment leads to p53-independent enhanced antitumor effects. Cell Death Dis. 2013;4:e503.

117. Spano C, et al. Soluble TRAIL armed human MSC as gene therapy for pancreatic cancer. Sci Rep. 2019;9(1):1788.

118. Kolluri KK, Laurent GJ, Janes SM. Mesenchymal stem cells as vectors for lung cancer therapy. Respiration. 2013;85(6):443-51.

119. Kauer TM, et al. Encapsulated therapeutic stem cells implanted in the tumor resection cavity induce cell death in gliomas. Nat Neurosci. 2011;15(2):197-204.

120. Menon LG, et al. Human bone marrow-derived mesenchymal stromal cells expressing S-TRAIL as a cellular delivery vehicle for human glioma therapy. Stem Cells. 2009;27(9):2320-30.

121. Loebinger MR, et al. Mesenchymal stem cell delivery of TRAIL can eliminate metastatic cancer. Cancer Res. 2009;69(10):4134-42.

122. Sasportas LS, et al. Assessment of therapeutic efficacy and fate of engineered human mesenchymal stem cells for cancer therapy. Proc Natl Acad Sci USA. 2009;106(12):4822-7.

123. Duebgen $M$, et al. Stem cells loaded with multimechanistic oncolytic herpes simplex virus variants for brain tumor therapy. J Natl Cancer Inst. 2014;106(6):dju090.

124. Shah K. Mesenchymal stem cells engineered for cancer therapy. Adv Drug Deliv Rev. 2012;64(8):739-48.

125. Levy $\mathrm{O}$, et al. mRNA-engineered mesenchymal stem cells for targeted delivery of interleukin-10 to sites of inflammation. Blood. 2013:122(14):e23-32.

126. Kalimuthu S, et al. A new approach for loading anticancer drugs into mesenchymal stem cell-derived exosome mimetics for cancer therapy. Front Pharmacol. 2018;9:1116.

127. Chulpanova DS, et al. Application of mesenchymal stem cells for therapeutic agent delivery in anti-tumor treatment. Front Pharmacol. 2018;9:259.

128. Cocce $V$, et al. Drug loaded gingival mesenchymal stromal cells (GinPa-MSCs) inhibit in vitro proliferation of oral squamous cell carcinoma. Sci Rep. 2017;7(1):9376.

129. Sun L, et al. Mesenchymal stem cells functionalized sonodynamic treatment for improving therapeutic efficacy and compliance of orthotopic oral cancer. Adv Mater. 2020;32(48):e2005295.

130. Nawaz $M$, et al. The emerging role of extracellular vesicles as biomarkers for urogenital cancers. Nat Rev Urol. 2014;11(12):688-701.

131. Wu H, et al. Bone marrow mesenchymal stem cells-derived exosomal microRNA-193a reduces cisplatin resistance of non-small cell lung cancer cells via targeting LRRC1. Cell Death Dis. 2020;11(9):801.

132. Munoz JL, et al. Delivery of functional anti-miR-9 by mesenchymal stem cell-derived exosomes to glioblastoma multiforme cells conferred chemosensitivity. Mol Ther Nucleic Acids. 2013;2:e126. 
133. Li H, et al. Exosomes derived from siRNA against GRP78 modified bonemarrow-derived mesenchymal stem cells suppress Sorafenib resistance in hepatocellular carcinoma. J Nanobiotechnol. 2018;16(1):103.

134. Lou G, et al. Exosomes derived from miR-122-modified adipose tissuederived MSCs increase chemosensitivity of hepatocellular carcinoma. J Hematol Oncol. 2015;8:122.

135. Zhou Y, et al. Bone marrow mesenchymal stem cells-derived exosomes for penetrating and targeted chemotherapy of pancreatic cancer. Acta Pharm Sin B. 2020;10(8):1563-75.

136. Rubio D, et al. Spontaneous human adult stem cell transformation. Cancer Res. 2005:65(8):3035-9.

137. de la Fuente R, et al. Retraction: spontaneous human adult stem cell transformation. Cancer Res. 2010;70(16):6682.

138. Ben-David U, Mayshar Y, Benvenisty N. Large-scale analysis reveals acquisition of lineage-specific chromosomal aberrations in human adult stem cells. Cell Stem Cell. 2011:9(2):97-102.

139. Tarte $K$, et al. Clinical-grade production of human mesenchymal stromal cells: occurrence of aneuploidy without transformation. Blood. 2010;115(8):1549-53.

140. von Bahr $\mathrm{L}$, et al. Analysis of tissues following mesenchymal stromal cell therapy in humans indicates limited long-term engraftment and no ectopic tissue formation. Stem Cells. 2012;30(7):1575-8.

141. Barkholt $L$, et al. Risk of tumorigenicity in mesenchymal stromal cell-based therapies-bridging scientific observations and regulatory viewpoints. Cytotherapy. 2013;15(7):753-9.

142. Fregni $G$, et al. Reciprocal modulation of mesenchymal stem cells and tumor cells promotes lung cancer metastasis. EBioMedicine. 2018;29:128-45

143. Xu G, et al. Adenoviral-mediated interleukin-18 expression in mesenchymal stem cells effectively suppresses the growth of glioma in rats. Cell Biol Int. 2009;33(4):466-74.

144. Kanehira M, et al. Targeted delivery of NK4 to multiple lung tumors by bone marrow-derived mesenchymal stem cells. Cancer Gene Ther. 2007;14(11):894-903.

145. Ren C, et al. Cancer gene therapy using mesenchymal stem cells expressing interferon-beta in a mouse prostate cancer lung metastasis model. Gene Ther. 2008;15(21):1446-53.
146. LiX et al. In vitro effect of adenovirus-mediated human Gamma Interferon gene transfer into human mesenchymal stem cells for chronic myelogenous leukemia. Hematol Oncol. 2006;24(3):151-8.

147. Xin $\mathrm{H}$, et al. Targeted delivery of CX3CL1 to multiple lung tumors by mesenchymal stem cells. Stem Cells. 2007;25(7):1618-26.

148. Stuckey DW, et al. Engineering toxin-resistant therapeutic stem cells to treat brain tumors. Stem Cells. 2015:33(2):589-600.

149. Hu M, et al. Anti-angiogenesis therapy based on the bone marrowderived stromal cells genetically engineered to express sFlt-1 in mouse tumor model. BMC Cancer. 2008;8:306.

150. Xiang J, et al. Mesenchymal stem cells as a gene therapy carrier for treatment of fibrosarcoma. Cytotherapy. 2009;11(5):516-26.

151. Choi SA, et al. Human adipose tissue-derived mesenchymal stem cells: characteristics and therapeutic potential as cellular vehicles for prodrug gene therapy against brainstem gliomas. Eur J Cancer. 2012:48(1):129-37.

152. Lang FM, et al. Mesenchymal stem cells as natural biofactories for exosomes carrying miR-124a in the treatment of gliomas. Neuro Oncol. 2018;20(3):380-90.

153. Shimbo K, et al. Exosome-formed synthetic microRNA-143 is transferred to osteosarcoma cells and inhibits their migration. Biochem Biophys Res Commun. 2014:445(2):381-7.

154. Katakowski $M$, et al. Exosomes from marrow stromal cells expressing miR-146b inhibit glioma growth. Cancer Lett. 2013;335(1):201-4.

155. Chen Z, et al. microRNA-6785-5p-loaded human umbilical cord mesenchymal stem cells-derived exosomes suppress angiogenesis and metastasis in gastric cancer via INHBA. Life Sci. 2021;284:

\section{Publisher's Note}

Springer Nature remains neutral with regard to jurisdictional claims in published maps and institutional affiliations.
Ready to submit your research? Choose BMC and benefit from:

- fast, convenient online submission

- thorough peer review by experienced researchers in your field

- rapid publication on acceptance

- support for research data, including large and complex data types

- gold Open Access which fosters wider collaboration and increased citations

- maximum visibility for your research: over $100 \mathrm{M}$ website views per year

At $\mathrm{BMC}$, research is always in progress.

Learn more biomedcentral.com/submissions 